\title{
DISCUSSION FOLLOWING PAPER BY ARNETT
}

Fowler to Schramm: What is the atomic mass of the fourth peak which your last graph showed? Does it reach into the region of the superheavy elements?

Schramm: It is near atomic mass 281 and the neutron magic number 184 and is therefore not composed of truly superheavy nuclei.

Fowler: I wish to emphasize the point raised by Dr Arnett in his excellent presentation of nuclear processes in the late stages of stellar evolution - the extrapolation of the $S$-factor to low energies in the heavy ion reactions ${ }^{12} \mathrm{C}+{ }^{12} \mathrm{C},{ }^{12} \mathrm{C}+{ }^{16} \mathrm{O}$ and ${ }^{16} \mathrm{O}+{ }^{16} \mathrm{O}$ is very uncertain! Michaud claims that $S$ continues to rise at low energy due to reactions under the coulomb barrier. On the other hand the rise at the lowest observable energies may be a 'shape-resonance' and may be followed at still lower energies by a marked decrease. Work at Caltech on the reaction ${ }^{16} \mathrm{O}+{ }^{28} \mathrm{Si}$ is attempting to prove or disprove Michaud's effect. It will be some time before the experimentalists can resolve the issue.

Hesser to Arnett: Perhaps the study of HR diagrams will yet be able to provide some useful constraints for the theories which you, and others, have been discussing, but to push the observations to such a point will require much hard work. For instance, with the new large reflectors in the southern hemisphere, it may be possible to study the luminosity function of stars on their way to becoming white dwarfs in the nearest globular clusters.

As an example of the type of work that may be done when we leave aside our classical notions that all stars in clusters must lie on 'sequences' we have the beautiful work of Zinn and Norris at Yale. They are studying those stars that lie to the left of the giant branch and above the horizontal branch.

The difficulty of finding candidate stars (many clusters must be searched) and of obtaining the subsequent data should not discourage us in our attempts to use the observed HR diagram, with its many unexplained nuances, as valuable guide for theory in the future. 